\title{
Impianti di depurazione delle acque reflue: progettazione di sistemi di disinfezione
}

\author{
Aurelio Striano ${ }^{1}$ \\ ${ }^{1}$ Affiliation not available
}

\begin{abstract}
La disponibilità e qualità delle risorse costituiscono condizione essenziale per il sostentamento di tutte le forme di vita animale e vegetale, secondo un complesso sistema di equilibri tra produzione, utilizzazione e accumulo.

L'uomo ha fondato il proprio sviluppo socio - economico sulla disponibilità e qualità delle risorse naturali, accrescendo in modo eccezionale la capacità di utilizzarle e modificarle in tempi molto brevi, specie se confrontati con i tempi di crescita delle risorse naturali. Da ciò consegue il rischio di provocare una significativa riduzione della disponibilità.

Tale problematica, riguarda soprattutto la principale risorsa che l'uomo ha avuto per prima a disposizione, sin dalla sua nascita, ossia l'acqua.

È necessario, pertanto, individuare sistemi che possano permettere il riutilizzo di tale risorsa in una forma che non possa nuocere alla salute dell'uomo.

È per tale motivo che assume particolare importanza il tema della disinfezione delle acque, attraverso metodologie convenzionali, ma ancor di più, attraverso metodologie avanzate e innovative, le quali, oltre ad essere estremamente efficaci per la disinfezione delle acque, sono anche molto efficienti sotto l'aspetto dell'inquinamento, che negli ultimi tempi sta acquistando sempre più importanza.
\end{abstract}




\section{Introduzione}

La problematica della scarsità dell' acqua, spesso, è affiancata dalla problematica della sua disponibilità in una forma "non pulita", ossia non depurata da inquinanti organici e/o inorganici. Quando tali microrganismi non sono rimossi dall' acqua, l'uso della stessa causa il proliferare di malattie e in casi più estesi anche un possibile sviluppo di epidemie.

Molte attività umane portano alla produzione di scarichi, chiamati acque reflue che, al fine di poter essere restituite all'ambiente, devono essere oggetto di depurazione, poiché la quantità di sostante inquinanti è superiore alla capacità auto-depurativa di terreni, mari, fiumi e laghi.

Attraverso le caratteristiche quantitative delle acque reflue è possibile fare una classificazione di esse in (Fig. 1):

- acque nere, provenienti dalla rete di distribuzione o da prelievi diretti, per l'uso civile e delle attività produttive e sono costituite da: acque di scarico delle case, acque saponate grasse (con olio e detersivi scaricati dalle cucine), oppure da acque di scarico industriale;

- acque bianche, costituite da acque di pioggia e da acque che si infiltrano nelle fogne dalla falda sotterranea.

Il trattamento di depurazione dei liquami urbani consiste in una successione di più fasi (o processi) durante i quali vengono rimosse le sostanze indesiderate dall'acqua reflua. Tale processo consiste dunque nella disattivazione, rimozione, o uccisione dei microrganismi patogeni presenti, attraverso una serie di trattamenti che hanno come scopo la conseguente fine del loro sviluppo e della loro riproduzione.

La disinfezione è solitamente la fase finale del processo di trattamento delle acque al fine di limitare 
gli effetti del materiale organico, dei solidi sospesi e di altri contaminanti.

Le acque vengono poi concentrate, dando luogo a un effluente, compatibile con la capacità autodepurativa del corpo ricettore prescelto per lo sversamento, senza che questo ne possa subire danni.

\section{Confronto diretto tra i due impianti: i pro e contro di entrambi}

Tra le varie metodologie di disinfezione esistenti è stata posta l'attenzione sulla disinfezione delle acque bastata sull'utilizzo del cloro, dunque attraverso metodologie basate sull'utilizzo di sostanze chimiche e sulla disinfezione delle acque basata sull'utilizzo dei raggi UV, dunque attraverso metodologie basate sull'utilizzo delle radiazioni ultraviolette.

Il meccanismo di azione delle radiazioni ultraviolette è poco confrontabile con quello dei disinfettanti chimici, che agisce sulla denaturazione di macromolecole (lipidi, proteine) spesso protette da strutture interne ed esterne dei germi.

Però è possibile sottolineare sicuramente che l'aspetto che rende particolarmente interessante l'attività biocida dei raggi UV è che le dosi necessarie per uccidere microrganismi patogeni (inclusi i virus, le spore, le cisti di protozoi) sono molto più compatibili con l'ambiente di quanto non lo siano le concentrazioni del cloro.

Sia la disinfezione delle acque attraverso il cloro che la disinfezione delle acque attraverso i raggi UV utilizzano le migliori tecniche ad oggi disponibili. È possibile dunque mettere a confronto le due differenti tipologie per la disinfezione, cogliendone di entrambi i vantaggi e svantaggi.

Il vantaggio principale della clorazione (Fig. 2) è sicuramente quello del processo più utilizzato 
per la depurazione microbiologica delle acque. Esso reagisce ossidando le sostanze organiche e inorganiche e inattivando i microrganismi.

Tuttavia, il cloro può reagire con il materiale organico disciolto e produrre alcune sostanze chimiche tossiche come il triclorometano (cloroformio) che possono essere collegate a rischi per la salute umana. Inoltre, contro microrganismi parassiti, quali Cryptosporidium e Giardia, la clorazione al dosaggio massimo consentito risulta inefficace.

Il vantaggio principale della disinfezione attraverso raggi UV (Fig. 3) è sicuramente quella di non utilizzare sostanze chimiche come il cloro né di lasciare prodotti nocivi. Alcuni sistemi UV non richiedono l'impiego di personale specializzato, avendo manutenzione ridotta: basta cambiare la lampadina UV ogni anno.

Altre volte invece lo sterilizzatore UV può aver bisogno di interventi manutentivi, altrimenti può venir meno l'effetto sterilizzante. Per lo più è importante rimuovere il calcare che si accumula sul tubo di quarzo che forma una patina che impedisce ai raggi ultravioletti di penetrare. La pulizia si esegue con appositi prodotti. Non destano preoccupazioni derivanti da danni per sovradosaggio o da incidenti, non danneggiano gli impianti idraulici. Sono sistemi modulabili e adattabili ai molteplici corpi idrici da trattare.

Di contro uno sterilizzatore a ultravioletti, non alterando le caratteristiche chimico fisiche, non può assolutamente potabilizzare un'acqua non potabile. Questo perché la radiazione UV è efficace solo per il trattamento di batteri e virus. È importantissimo che l'acqua sia pura, poiché le particelle possono fare ombra e proteggere i microorganismi che si trovano dietro. 


\section{Conclusione}

Tutte le metodologie del sistema di disinfezione attraverso i raggi UV e tutte le loro possibili combinazioni sono un mezzo, oltre che molto efficace per la disinfezione dell'acqua, anche molto efficiente riguardo la tematica dell'inquinamento, che negli ultimi tempi sta acquistando sempre importanza. Infatti, in un'ottica di un processo di depurazione che miri ad uno sviluppo sostenibile, gli impianti per la disinfezione delle acque attraverso l'utilizzo di raggi UV rappresentano una tecnologia promettente per il trattamento avanzato delle acque reflue.

Facendo poi un discorso attuale e di grandissima importanza, un po' più generale, non solo legato alla tematica della disinfezione delle acque, la pandemia COVID-19, in corso in tutto il mondo, ha portato ad un aumento della domanda di sistemi automatizzati di disinfezione delle superfici basati sulla luce UV-C. La richiesta è stata sollevata soprattutto dall'industria sanitaria e dalle istituzioni commerciali per fermare la trasmissione del virus evitando le pratiche di pulizia manuale. Questo fattore dovrebbe guidare la crescita del mercato delle apparecchiature di disinfezione UV in generale, dunque non solo di quelle legate alla disinfezione dell'acqua.

Il mercato globale delle apparecchiature di disinfezione UV è stato valutato a $\$ 1,3$ miliardi nel 2019 e si prevede che raggiungerà \$ 5,7 miliardi entro il 2027, crescendo a un CAGR del 17,1\% dal 2020 al 2027. (Chaudhary A. et al., 2020).

Si prevede che gli elevati investimenti in ricerca e sviluppo da parte dei produttori per migliorare le prestazioni delle apparecchiature di disinfezione UV per applicazioni di grandi volumi come l'acqua potabile municipale e il trattamento delle acque di processo industriali guideranno la crescita del mercato 


\section{References}

1.Angelini L., A. S., Guidi E. Popolazione e Storia. 53-67 (2003).

2.Babaei A.A., G. F. COD removal from petrochemical wastewater by UV / hydrogen peroxide UV / persulfate and UV / percarbonate: biodegradability improvement and cost evaluation.. 484-494 (2016).

3.Anipsitakis G.P., D. D. D. Degradation of organic contaminants in water with sulfate radicals generated by the conjunction of peroxymonosulfate with cobalt.. 4790-4797 (2003).

4.Barreiro R., P. J. R. Toxic effects of chemicals on microorganisms, Environ.. 632 (1992).

5.Bertanza G., C. C. Pre-treatment of industrial waste: comparison between the Fenton Process and the Alternative Oxidation processes.. (2000).

6.Bhattacharjee S., S. Y. T. Mechanisms for advanced photooxidation of aqueous organic waste compounds.. 1 (1998).

7.C., C. Advanced Oxidation Technologies, Boulevard de la Woluwe.. (1997).

8.Y., D. Advanced oxidation processes (AOPs) for reduction of organic pollutants in landfill leachate.. 366-384 (2009).

9.Indicator compounds for assessment of wastewater effluent contributions to flow and water quality.. 1199-1212 (2011).

10.H.J.H., F. Oxidation of tartaric acid in the presence of iron.. 899 (1894).

11.S., G. A review of the concepts, recent advances and niche applications of the (photo) Fenton process, beyond water / wastewater treatment: Surface functionalization, biomass treatment, combatting cancer and other medical uses.. 309-319 (2019). 
12.Glaze W.H., C. D. H., Kang J.W. The chemistry of water treatment processes involving ozone, hydrogen peroxide and ultraviolet radiation.. 355 (1987).

13.Haber F., W. J. The catalytic decomposition of hydrogen peroxide by iron salts. 332 (1934).

14.Stabilized landfill leachate treatment by coagulation - flocculation coupled with UV-based sulfate radical oxidation process.. 575-581 (2018).

15.Jyoti K.K., P. A. B. Water disinfection by acoustic and Hydrodinamic cavitation.. 201-212 (2001).

16.Beneficial effect of homogeneous photo-Fenton pretreatment upon the biodegradation of anthraquinone sulfonate in wastewater treatment.. 85 (1993).

17.Impact of water matrix on the removal of micropollutants by advanced oxidation technologies.. 155-173 (2019).

18.Legrini O., B. A. M., Oliveros E. Photochemical processes for water treatment.. 671 (1993).

19.Photochemical decomposition of 2,4-dichlorophenoxyacetic acid (2,4 - D) in aqueous solution. Part II.. (1996).

20.L., M. Depurazione delle acque. Tecniche ed impianti per il trattamento delle acque di rifiuto.. (2012).

21.Ollis D., A.-E. H. Photocatalytic Purification of Water and Air.. (1993).

22.J.J., P. Dark and photoassisted Fe3+ — catalyzed degradation of chlorophenoxy herbicides by hydrogen peroxide.. 944 (1992).

23.Peyton G.R., G. W. H. Destruction of pollutants in water with ozone in combination with ultraviolet radiation. Part 3. Photolysis of aqueous ozone. Mechanism of photolytic ozonation.. 761 (1988). 
24.Degradation of 2,4-dichlorophenoxyacetic acid by ozone and light.. 235 (1994).

25.Pulgarin C., K. J. Overview on photocatalytic and electrocatalytic pretreatment of industrial non-biodegradable pollutants and pesticides.. 50 (1996).

26.Verlicchi P., M. L. Sistemi "convenzionali" e sistemi "naturali” di disinfezione delle acque reflue.. 2-8 (2005). 


\section{Figure Captions}

Figure 1. Raffigurazione di acque nere e bianche (Fonte: chem-service.it)

Figure 2. Impianto per la disinfezione delle acque tramite clorazione

Figure 3. Impianto per la disinfezione delle acque tramite raggi UV 


\section{Figures}

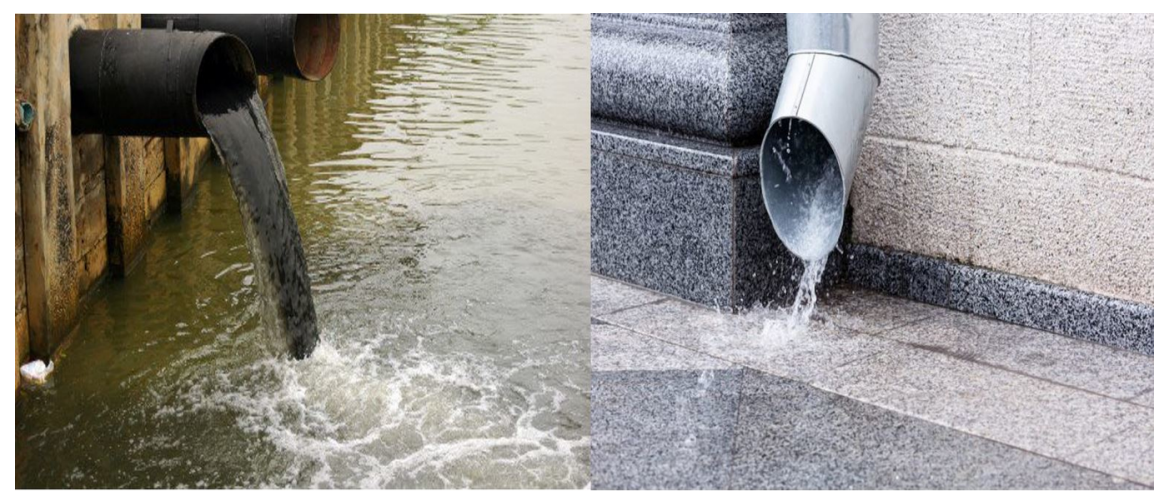

Figure 1: Raffigurazione di acque nere e bianche (Fonte: chem-service.it)

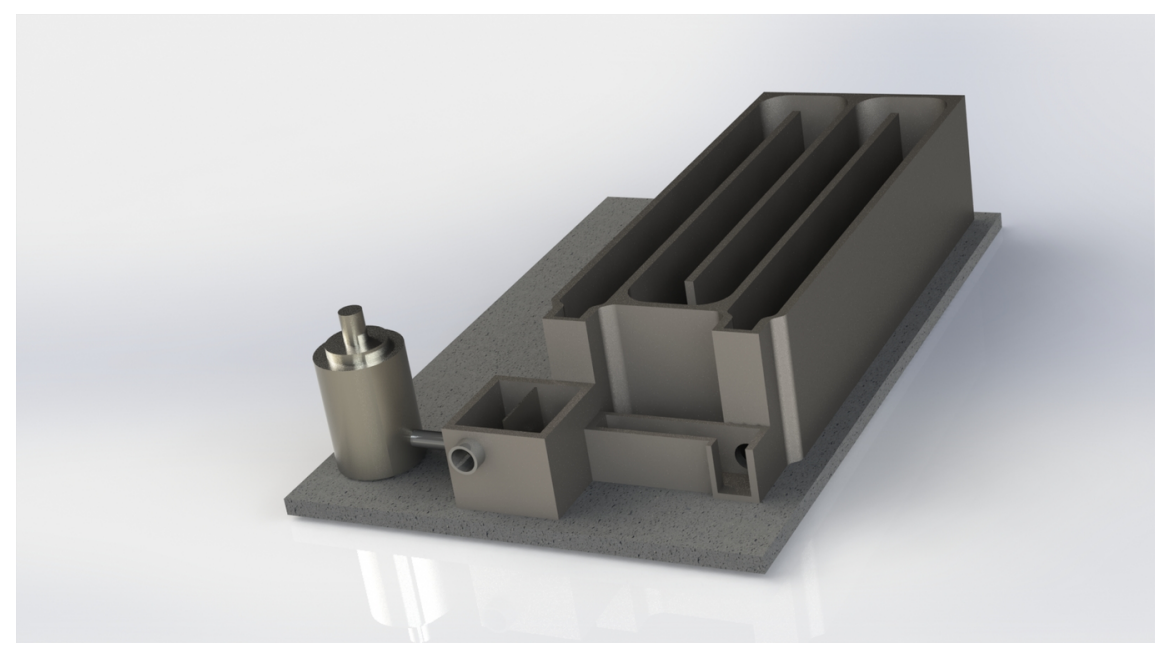

Figure 2: Impianto per la disinfezione delle acque tramite clorazione 


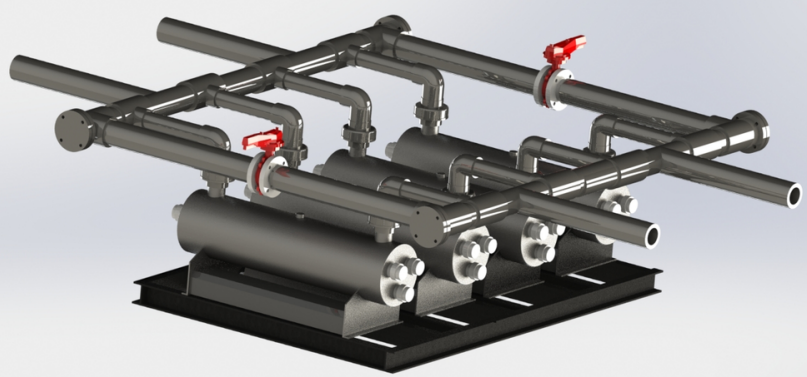

Figure 3: Impianto per la disinfezione delle acque tramite raggi UV 